\title{
Experimental Study of Heat Flux Distribution of Arc Driven by AC Magnetic Field
}

\author{
Naomi Matsumoto, Takeo Yamamoto, Masaya Sugimoto, and Koichi Takeda \\ Department of Machine Intelligence and System Engineering, Akita Prefectural University, Yurihonjo 015-0055, Japan \\ Correspondence should be addressed to Masaya Sugimoto, sugimoto@akita-pu.ac.jp
}

Received 3 December 2010; Accepted 21 February 2011

Academic Editor: Pavel Lejcek

Copyright () 2011 Naomi Matsumoto et al. This is an open access article distributed under the Creative Commons Attribution License, which permits unrestricted use, distribution, and reproduction in any medium, provided the original work is properly cited.

\begin{abstract}
The authors earlier developed a model to predict the heat flux distribution in an arc driven by an AC magnetic field. That theoretical model implied that the heat flux distribution depended on waveforms of the imposed AC magnetic field. Experiments were conducted in this study to validate that theoretical prediction. Theoretical calculations of heat flux distribution in the arc driven by AC magnetic field were conducted using the heat flux profile in the arc root obtained from the measurement under no magnetic field. The heat flux distributions in arcs driven by AC magnetic fields were measured by imposing two AC magnetic fields with sinusoidal and rectangular waveforms. Agreement between experimental and theoretical heat flux distributions was good. Results confirm that heat flux profiles of various types are producible by controlling the imposed magnetic flux density and its waveform.
\end{abstract}

\section{Introduction}

Because of intense heat flow at a small arc root, transferred arcs have been widely used for cutting and welding of metals. However, conventional arcs remain inconvenient for heating and melting over a wide area. Many attempts have been made to expand arc roots using external magnetic fields [1, 2]. Takeda [3] developed a magnetically driven arc by imposing an alternating magnetic field perpendicular to a transferred arc. In the oscillatory motion of such a magnetically driven arc, the amplitude increases concomitantly with the increase of the imposed magnetic flux density. The oscillating arc can be regarded as a broad heat source with the width of oscillation amplitude. Practical applications of such a magnetically driven arc often necessitate precise understanding of the heat flux distribution. In a previous study [4], the variation of heat flux from an arc with magnetic flux density was considered theoretically under a DC magnetic field imposed perpendicular to the arc, where the distribution function of the heat flux in the arc root was assumed as triangular and one-dimensional. A schematic of the setup used to produce a magnetically driven arc is presented in Figure 1. Figure 2 shows that the arc root profile under no magnetic field is assumed as a circle of diameter $d_{0}$. When the magnetic field is imposed, the arc root shape deforms from a circle to an ellipse with major diameter $d_{Y}$ and minor diameter $d_{0}$. Consequently, the heat flux density in the arc root decreases concomitantly with increased magnetic flux density.

The authors recently developed a simple but practical model of a Gaussian function to predict the distributions of the heat flux density under various waveforms of the imposed AC magnetic field [5]. In the present work, experimentation was used to confirm the theoretical predictions.

\section{Theoretical Expression of the Time Averaged Heat Flux Distribution of the Arc Driven by an AC Magnetic Field}

The heat flux profile in the arc root on the anode under no magnetic field is assumed to be expressed using a Gaussian distribution function as

$$
H_{0}(y)=\frac{P_{0}}{\sigma_{0} \sqrt{2 \pi}} \exp \left(-\frac{y^{2}}{\sigma_{0}^{2}}\right) .
$$

Therein, $P_{0}$ and $\sigma_{0}$, respectively, signify the total heat flow from the arc to the anode and a measure of the width of the Gaussian distribution function. When the DC magnetic 


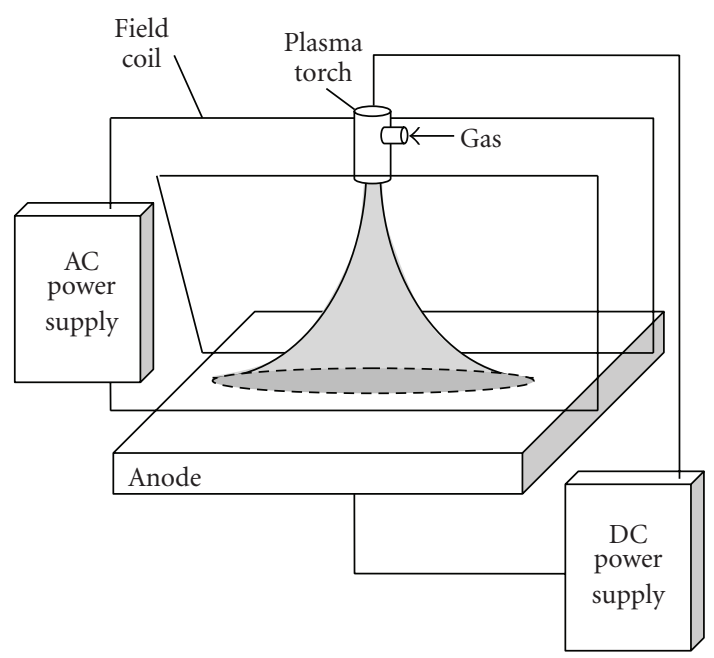

Figure 1: Schematic illustration of a system used to produce a magnetically driven arc.

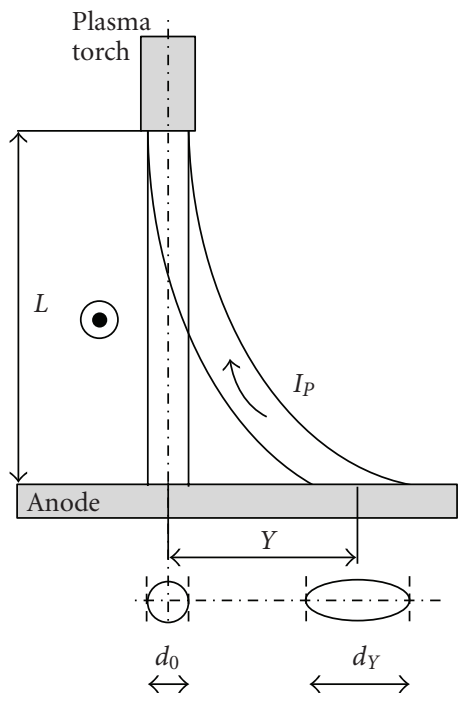

FIgURE 2: Schematic illustration on the arc root deformation by an imposed DC magnetic field.

field is imposed on the arc, the heat flux profile, whose center shifts to $Y$, deforms as

$$
H_{Y}(y)=\frac{P_{0}}{\sigma_{Y} \sqrt{2 \pi}} \exp \left(-\frac{(y-Y)^{2}}{\sigma_{Y}^{2}}\right) .
$$

In that equation, $\sigma_{0}$ and $\sigma_{Y}$ satisfy the relation presented below:

$$
\frac{\sigma_{Y}}{\sigma_{0}}=\frac{d_{Y}}{d_{0}}=\frac{L^{2}+Y^{2}}{L^{2}-Y^{2}}
$$

where $L$ represents standoff distance between the torch and the anode. Typically, variations in the heat flux profile in the arc root with the increase of $Y$ are depicted as shown in Figure 3.

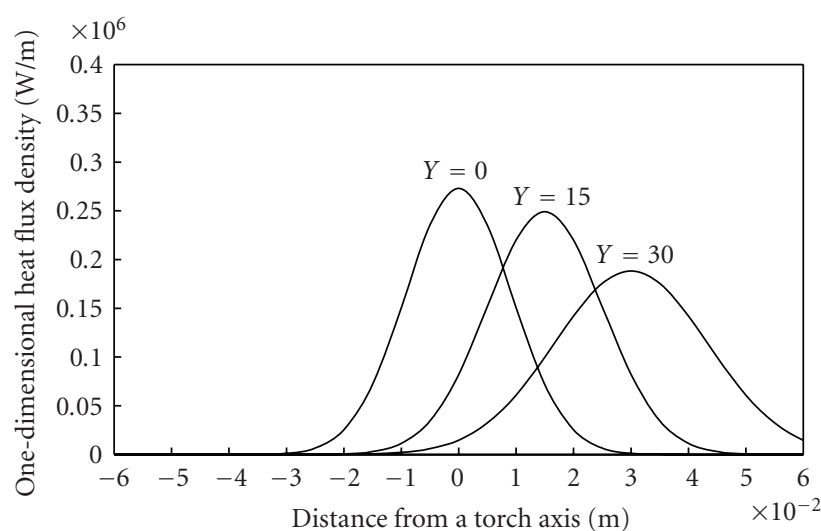

Figure 3: Typical variations in the heat flux profile in the arc root with increase of $Y$.

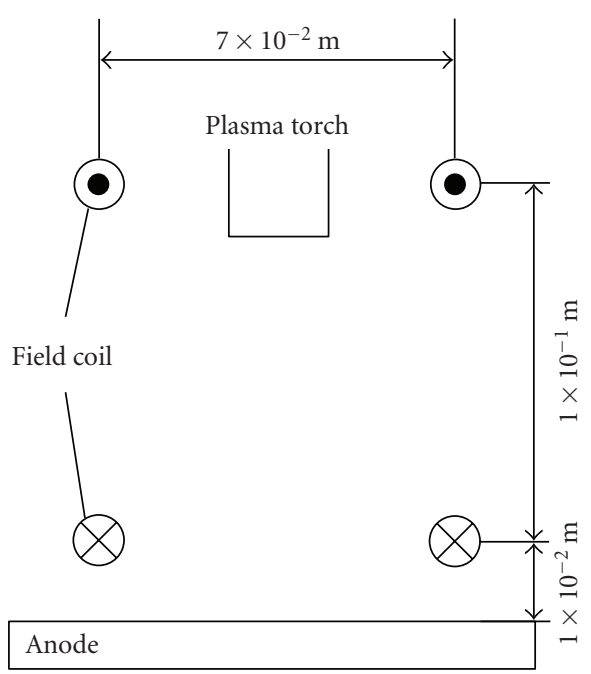

FIGURE 4: Dimensions of the magnetic field coil.

If the imposed $A C$ magnetic flux density $B(t)$ is expressed in the following arbitrary form as

$$
B(t)=B_{0} f(t)
$$

then the arc root center position $Y(t)$ can be reasonably assumed to have a similar form to that shown below:

$$
Y(t)=Y_{0} f(t) .
$$

Therein, $B_{0}$ and $Y_{0}$, respectively, represent the amplitudes of the AC magnetic flux density and the arc root movement. Function $f(t)$ represents the waveform varying between -1 and 1 . Then the heat flux averaged over the half period of the AC field is obtained as shown in

$$
\left\langle H_{Y_{0}}(y)\right\rangle=\frac{1}{\tau} \int_{-Y_{0}}^{Y_{0}} H_{Y}(y) \frac{d t}{d Y} d Y .
$$

In that equation, $\tau$ represents the half period of the imposed AC field. 


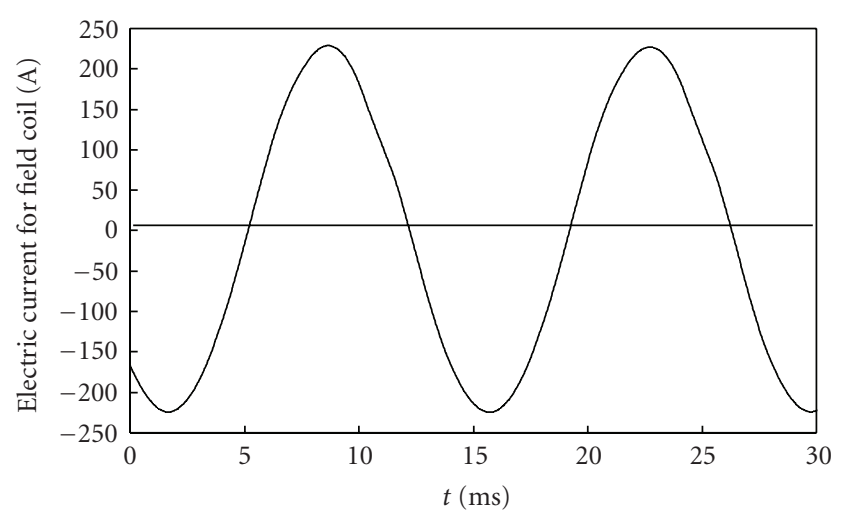

FIGURE 5: Coil current with sinusoidal waveform.

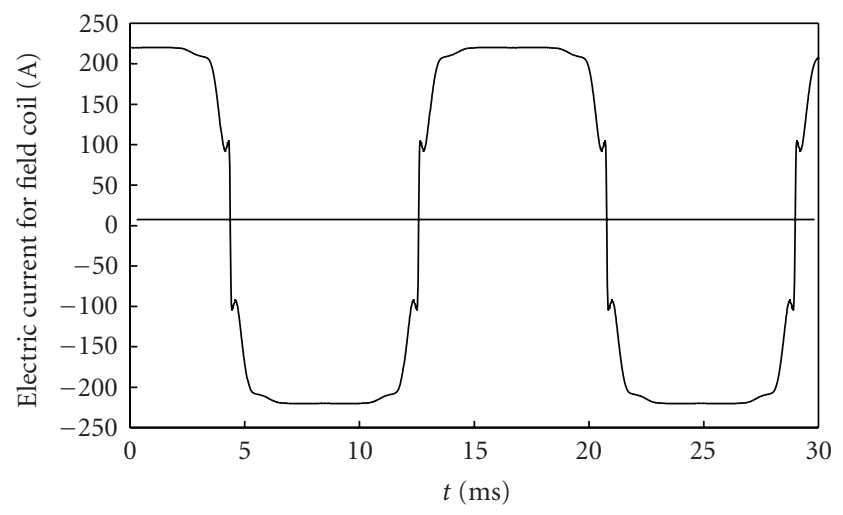

FIGURE 6: Coil current with rectangular waveform.

\section{Experiment}

Heat flux distributions in the arc driven by $\mathrm{AC}$ magnetic field were measured using the experimental arrangement presented schematically in Figure 1. A transferred arc was produced between a plasma torch with a tungsten cathode and a water-cooled copper anode. The standoff distance between the torch orifice and the anode was fixed at $7.0 \times$ $10^{-2} \mathrm{~m}$. A DC power supply connected to the torch and the anode was operated in a constant current mode at $80 \mathrm{~A}$. Its open circuit voltage was $280 \mathrm{~V}$. Argon was fed to the torch as a plasma forming gas at the flow rate of $4.5 \times 10^{-4} \mathrm{~kg} \mathrm{~s}^{-1}$. An external magnetic field was generated perpendicular to the arc using AC electric current through a two-turn rectangular field coil as shown in Figure 1. The coil arrangement on the $x-z$ plane is portrayed in Figure 4. The direction of the electric current through the upper two coil lines was inverse to that through the lower two coil lines. The maximum magnetic flux density in the coil center was $1.5 \times 10^{-3} \mathrm{~T}$ at the coil current of $250 \mathrm{~A}$. The AC magnetic field was produced by an $\mathrm{AC}$ power supply connected to the coil. In the experiment, the sinusoidal AC current and rectangular current were supplied to the coil. The frequency and amplitude of both currents were adjusted, respectively, at $70 \mathrm{~Hz}$ and $250 \mathrm{~A}$. Currents were measured using a clamp current meter. The

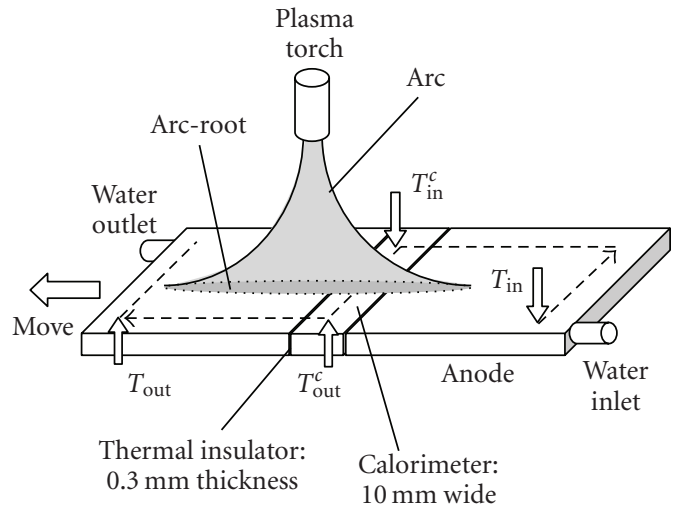

(a) Anode assembly and a calorimeter

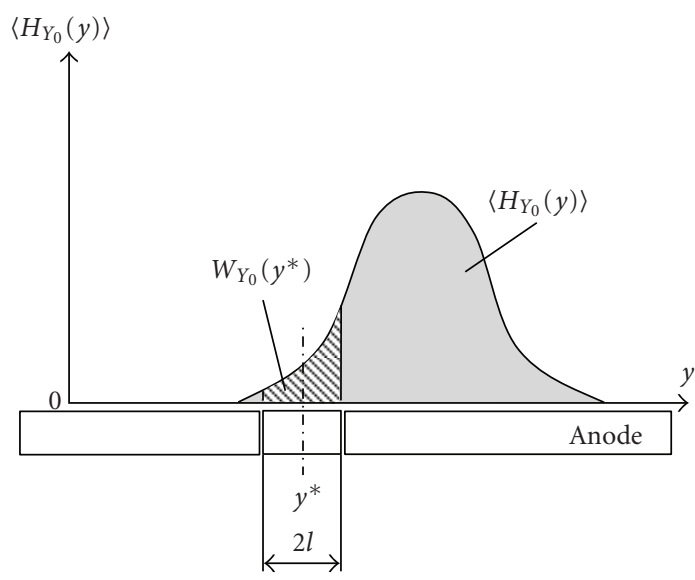

(b) Heat flow to the calorimeter

FIGURE 7: Anode assembly (a) and schematic representation of the heat flow to the calorimeter (b).

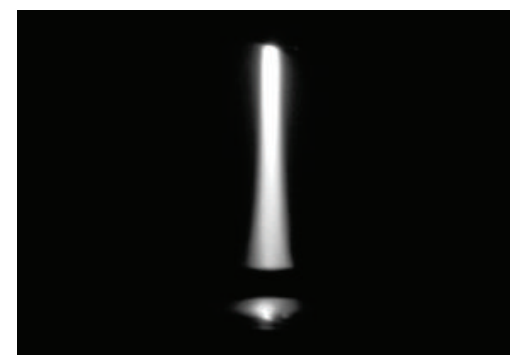

Figure 8: Plasma arc in no magnetic field.

different waveforms of currents used for these experiments are shown in Figures 5 and 6.

To observe the heat flux distribution in the arc driven by AC magnetic field, a special anode assembly was used. As Figure 7 shows, it consisted of three water-cooled copper blocks. The 10-mm-wide, 10-mm-thick, and 100-mm-long center block is designated hereinafter as a calorimeter. The calorimeter was thermally insulated from the others. Using a motor-drive mechanism, it was moved in the direction of the arc displacement at the velocity of $0.33 \mathrm{~mm} / \mathrm{s}$. The heat flow to the calorimeter was estimated from the temperature increase $\left(T_{\text {out }}^{C}-T_{\text {in }}^{C}\right)$ in the cooling water flowing through 


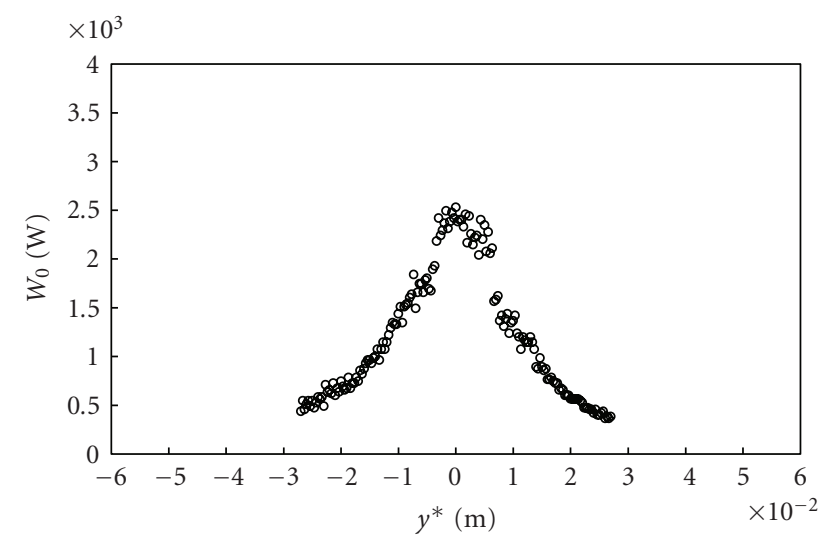

FIGURE 9: Variation of heat flow from the arc into the calorimeter in no imposed magnetic field.

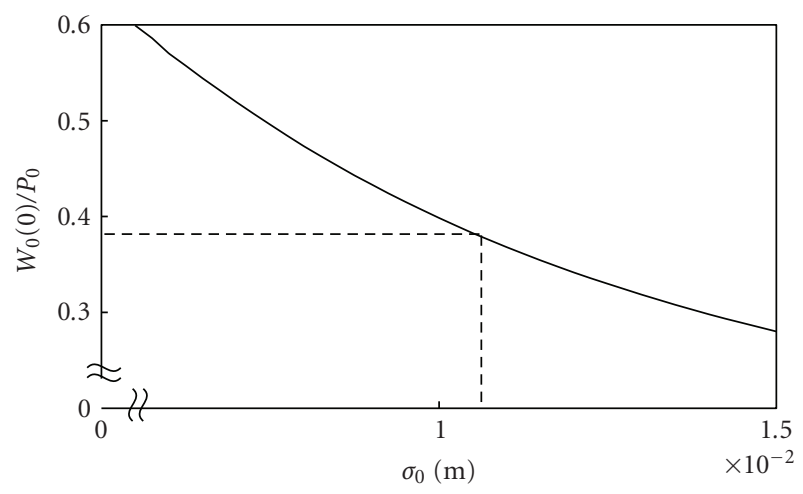

Figure 10: Theoretical variation of $W_{0}(0) / P_{0}$ with $\sigma_{0}$ and determination of the experimental value of $\sigma_{0}$.

this block. For temperature measurements, K-type thermocouples were used. Temperature variations were recorded at intervals of $1 \mathrm{~s}$. The total heat flow $P_{0}$ in (2) was determined from measurements of the temperature difference $\left(T_{\text {out }}-\right.$ $\left.T_{\text {in }}\right)$.

The distribution of the heat flux in the anode root can not be measured directly using a calorimeter. As portrayed in Figure $7(\mathrm{~b})$, the heat flow into the calorimeter having width of $2 l, W_{Y 0}\left(y^{*}\right)$ is expressed as

$$
W_{Y_{0}}\left(y^{*}\right)=\int_{y^{*}-l}^{y^{*}+l}\left\langle H_{Y_{0}}(y)\right\rangle d y,
$$

where $y^{*}$ and $\left\langle H_{Y}(y)\right\rangle$, respectively, represent the center position of the calorimeter and the time-averaged heat flux distribution represented by (6).

\section{Results and Discussion}

4.1. Determination of $H_{0}(y)$. Figure 8 portrays a snapshot of the arc with no imposed magnetic field. The stable arc was observed. Because of the pinch effect of the arc current, the arc column diameter was kept almost constant in the space between the torch and the anode. Considering the flow rate of $4.3 \times 10^{-2} \mathrm{~kg} / \mathrm{s}$ for the cooling water through the anode, the

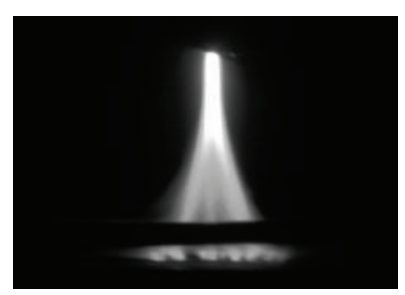

(a)

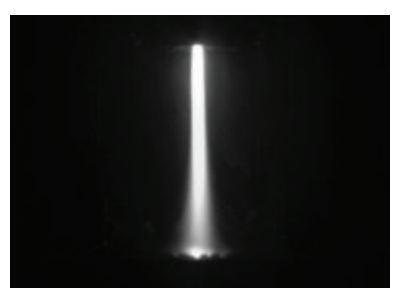

(b)
FIGURE 11: Arc driven by a sinusoidal AC magnetic field: front view (a) and side view (b).

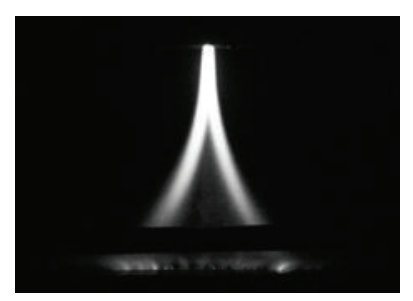

(a)

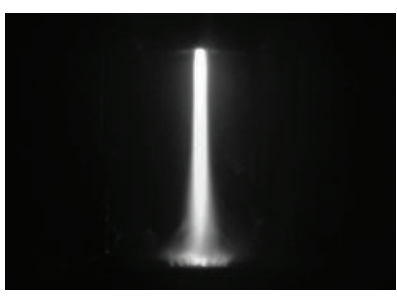

(b)
FIGURE 12: Arc driven by a rectangular AC magnetic field: front view (a) and side view (b).

measured temperature increase $\left(T_{\text {out }}-T_{\text {in }}\right)$ of $34 \mathrm{~K}$ provided the total heat flow from the arc to the anode as

$$
P_{0}=6.3 \mathrm{~kW}
$$

The obtained variation of the heat flow to the calorimeter from the stationary arc under no magnetic field is shown in Figure 9.

$$
\begin{aligned}
& \text { At } Y_{0}=0 \text { and } y^{*}=0,(7) \text { is represented as } \\
& \qquad W_{0}(0)=\int_{-l}^{+l} H_{0}(y) d y
\end{aligned}
$$

or

$$
\frac{W_{0}(0)}{P_{0}}=\frac{1}{\sigma_{0} \sqrt{2 \pi}} \int_{-l}^{+l} \exp \left(-\frac{y^{2}}{\sigma_{0}^{2}}\right) d y .
$$

Numerical calculation of the right-hand side of (10) as a function of $\sigma_{0}$ is illustrated in Figure 10. The obtained value of $W_{0}(0) / P_{0}$ from the experiment was 0.39 , which corresponds to the following in the figure:

$$
\sigma_{0}=1.1 \times 10^{-2} \mathrm{~m}
$$

4.2. Numerical Calculation of Heat Flux Distribution in the Arc Driven by an AC Magnetic Field. Snapshots of the arcs driven by a sinusoidal magnetic field and a rectangular one are portrayed, respectively, in Figures 11 and 12. Side views of the arc in both figures reveal that the arc moved only along the $y$ direction. From figures, the amplitudes of the arc motion are known in both cases as

$$
Y_{0}=2.2 \times 10^{-2} \mathrm{~m}
$$




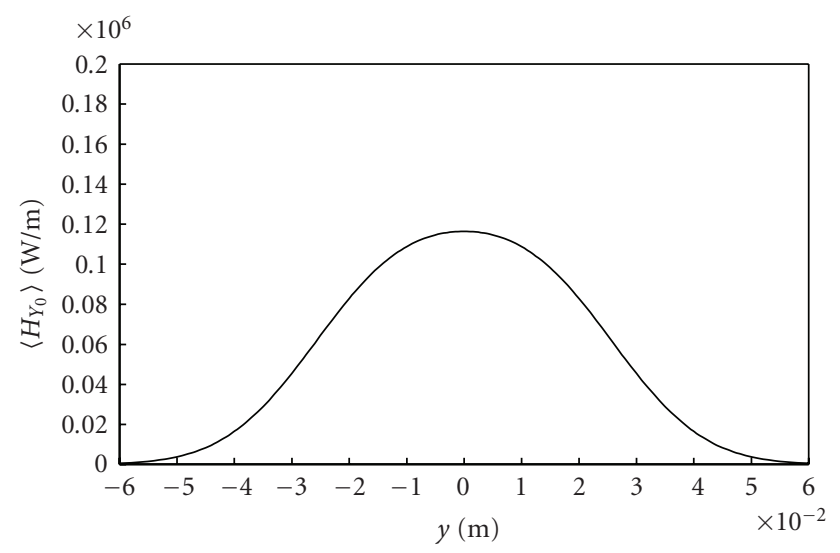

Figure 13: Heat flux distribution in the arc driven by a sinusoidal magnetic field. $Y_{0}$ is assumed as $2.2 \times 10^{-2} \mathrm{~m}$.

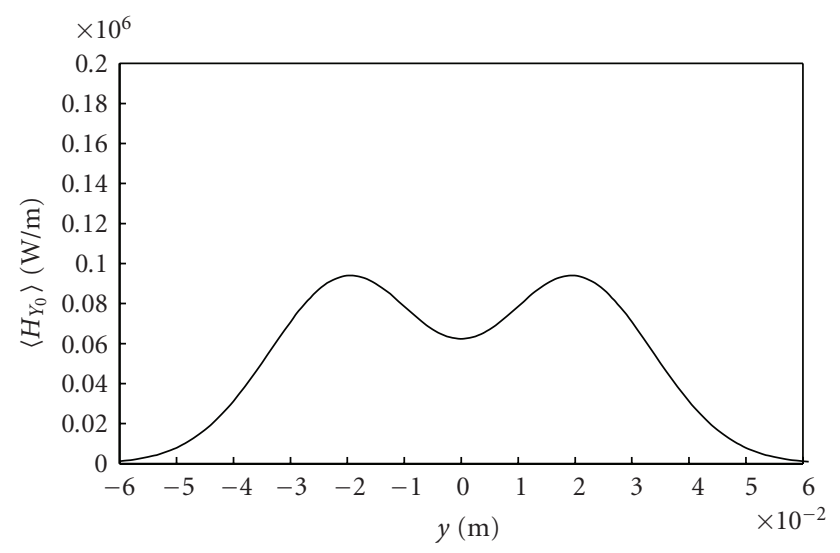

Figure 14: Heat flux distribution in the arc driven by a rectangular magnetic field. $Y_{0}$ is assumed as $2.2 \times 10^{-2} \mathrm{~m}$.

The heat flux distribution of (6) is calculable using obtained values of $P_{0}, \sigma_{0}$, and $Y_{0}$. The theoretical heat flux distribution in the arc driven by a sinusoidal magnetic field is presented in Figure 13; that by a rectangular one is portrayed in Figure 14.

\subsection{Comparison of Theoretical and Experimental Heat Flow} Distributions. Moving the calorimeter along the $y$ direction, the heat flow variations were measured under the AC magnetic fields of two types. As described in Section 3, the measurements were conducted not for the heat flux $\left\langle H_{Y_{0}}(y)\right\rangle$ but for the heat flow $W_{Y_{0}}\left(y^{*}\right)$. Experimental variations for the sinusoidal field and those for the rectangular one are shown, respectively, in Figures 15 and 16. In both figures, the theoretical variations of $W_{Y_{0}}\left(y^{*}\right)$ are shown as solid lines. Agreement between experimental results and theoretical predictions is good.

The arc output power is given by the product of the arc current and arc voltage. At the constant arc current of $80 \mathrm{~A}$, the arc output was maintained as $12 \mathrm{~kW}$ independent of the imposed magnetic field. Comparison of the arc output power with the total heat flow $P_{0}$ shows the efficiency of the heat transfer from the arc to the anode to be 53\%.

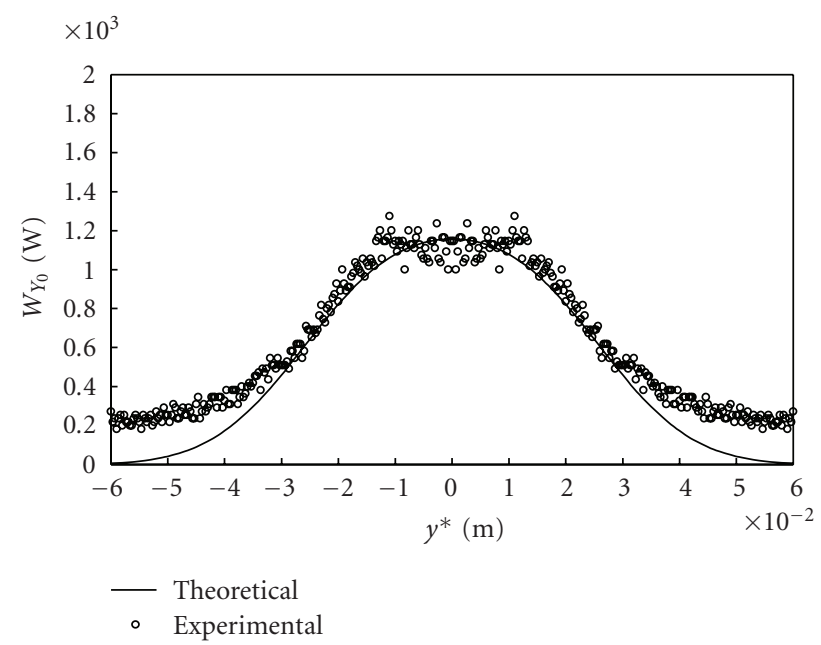

Figure 15: Heat flux distribution with a triangular magnetic field. $Y_{0}$ is assumed as $2.2 \times 10^{-2} \mathrm{~m}$.

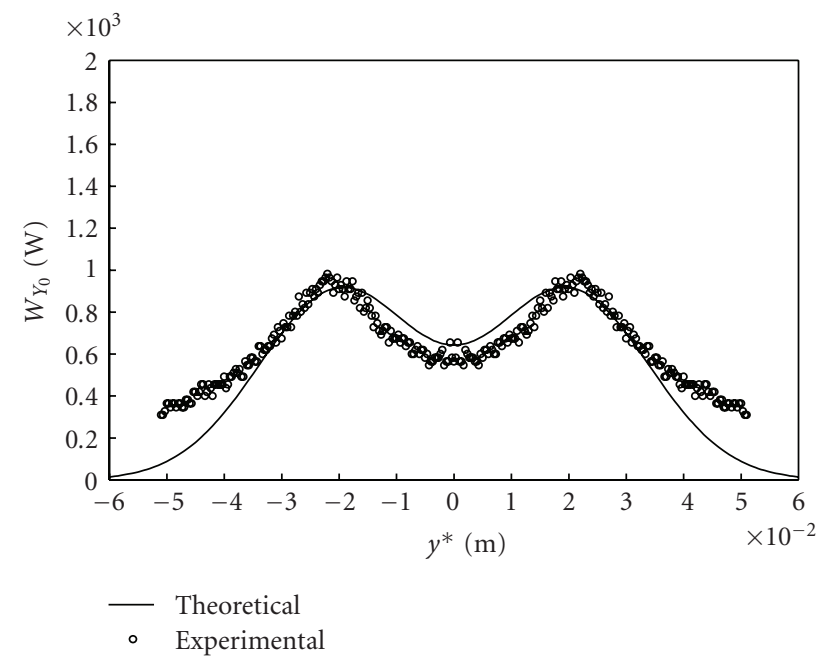

FIGURE 16: Heat flux distribution with a rectangular magnetic field. $Y_{0}$ is assumed as $2.2 \times 10^{-2} \mathrm{~m}$.

\section{Conclusion}

Earlier, the authors developed a model to predict the heat flux distribution in the arc driven by AC magnetic field. Results obtained using the theoretical model suggest that the heat flux distribution depended on the waveforms of the imposed AC magnetic field.

In this study, experiments were conducted to assess the validity of theoretical predictions. Theoretical calculations of heat flux distribution in the arc driven by AC magnetic field were conducted using the heat flux profile in the arc root obtained from the measurement under no magnetic field. The heat flux distributions in the arcs driven by AC magnetic fields were measured experimentally while imposing two AC magnetic fields having sinusoidal and rectangular waveforms. Agreement between the experimental and theoretical heat flux distributions was good. 
Results of the present study confirm that heat flux profiles of various types are producible by controlling the imposed magnetic flux density and its waveform.

\section{Acknowledgment}

This work was financially supported by Japan Science and Technology Agency.

\section{References}

[1] Y. Arata and H. Maruo, Technology Reports of the Osaka University, vol. 22, p. 135, 1972.

[2] J. E. Harry and D. Goodwin, "Surface heat treatment using a plasma torch with a magnetically traversed arc," in Proceedings of the 4th International Conference on Advances in Welding Processes, vol. 181, Harrogate, UK, May 1978.

[3] K. Takeda, "Generation of magnetically oscillating plasma arc and its properties," High Temperature Society, vol. 16, p. 357, 1990 (Japanese).

[4] N. Matsumoto, T. Usami, I. Kuno et al., "Heat flux of a transferred arc driven by a transverse magnetic field," Advances in Materials Science and Engineering, vol. 2009, Article ID 782048, 5 pages, 2009.

[5] T. Yamamoto, N. Matsumoto, M. Sugimoto, S. Sudo, T. Toh, and K. Takeda, "Theoretical consideration of heat flux distribution of arc driven by AC magnetic field," ISIJ International, vol. 49, no. 12, pp. 1845-1849, 2009. 

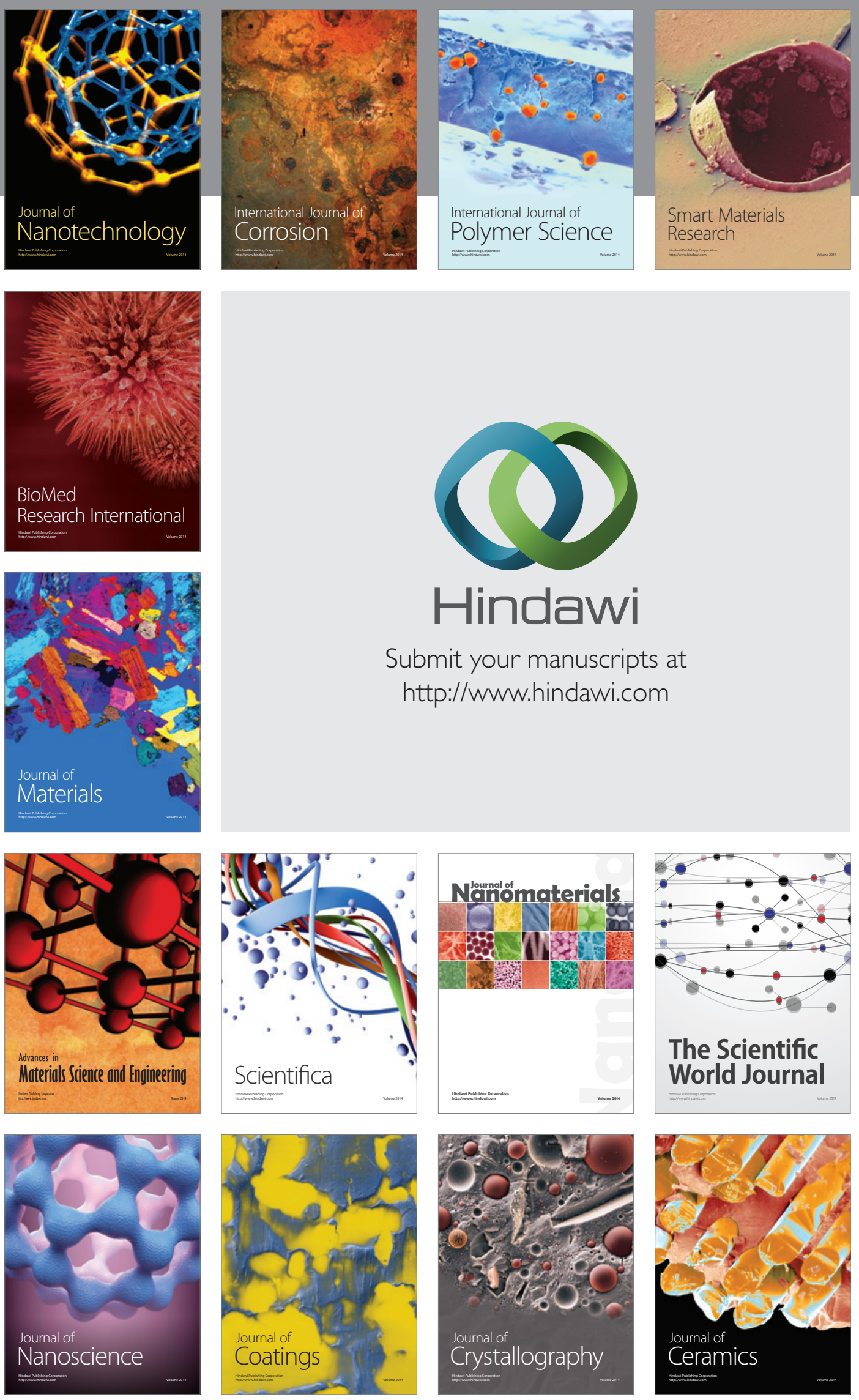

The Scientific World Journal

Submit your manuscripts at

http://www.hindawi.com

\section{World Journal}

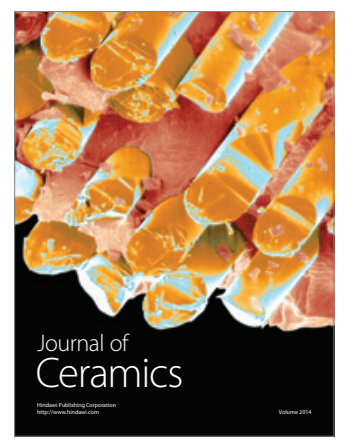

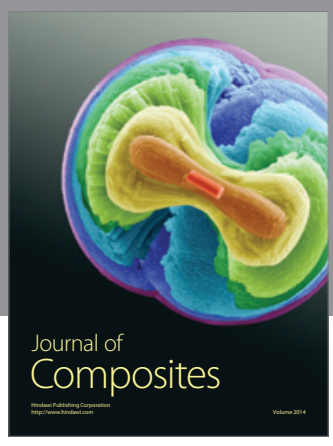
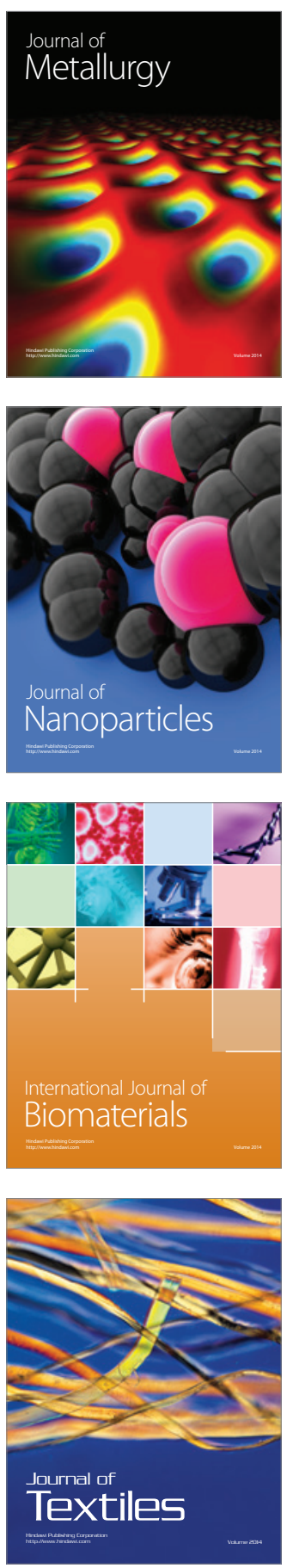